Article

\title{
Reversed biodegradation in cervical spine cartilage: The search for an acceptable way to provide the evidence for the hypothe- sis
}

Kirill V. Zhukov ${ }^{1}$, Alexandre A Vetcher ${ }^{1,2, *}$, Bagrat A. Gasparuan ${ }^{1}$ and Alexander Y. Shishonin ${ }^{1}$

${ }^{1}$ Complimentary and Integrative Health Clinic of Dr. Shishonin, 5 Yasnogorskaya Str, Moscow, 117588, Russian Federation; kirizhuk@yandex.ru (K.V.Z.), b.gasparyan@shishonin.ru (B.A.G), ashishonin@yahoo.com (A.Y.S)

2 Peoples' Friendship University of Russia (RUDN), 6 Miklukho-Maklaya St, 117198 Moscow, Russia

* Correspondence: avetcher@gmail.com

\begin{abstract}
If we accept that human body is a dissipative structure, then the recovery of the body should be considered as a redirection of the enegy flows. The recovery of vertebral cartilage through redirecting of inner dissipative flow requires the understanding of how is this case the fact of reversibility can be proven. We proposed the approach, that according to the collected data, satisfies all the scientific requirements.
\end{abstract}

Keywords: reversed biodegradation; arterial hypertension; vertebral cartilage

\section{Introduction}

The recovery of damaged vertebral cartilage is one of the challenges of modern medicine[1, 2]. The structure of cartilage (mostly extracellular matrix (65-80\% liquid content) dry part of which consists of collagens, proteoglycans, and in smaller amounts lipids, phospholipids, non-collagenous proteins, and glycoproteins [3]) and the ways of its natural decay allow considering this process as biodegradation[4]. Therefore, the process of non-invasive integrated recovery could be considered as reverse biodegradation with all the ensuing consequences.

In general, there are two approaches - surgery[1,5] and integrated treatment[6-8]. Nowadays there is a wide variety of techniques for the recovery from gene-based and cellbased therapies[9] to a multitude of polymer-based structures $[9,10]$ to replace or support the damaged part of the cartilage. Therefore, the issue of regeneration as an alternative to continuous degeneration eventually attracted the attention of the scientific community[11, 12]. The experiments are usually conducted on model animals like rats [12, 13] that makes it possible to measure some macro and molecular parameters of cartilages, analyzing separated tissues on different stages on the level of histological preparations.

The experimental set usually compares the averaged data since we could use the same animal only once, not the data from the same object on the different stages of recovery, that express the maximal interest. Indeed, only in this case, we will compare apples to apples. Therefore, we need to collect data from the same area of the same cartilage just on different stages of the cartilage recovery process without separation the cartilage for the histological staining. Moreover, the data, obtained from humans is significantly more valuable for Medicine. What methods give us the best opportunity?

From the very start let's list the most acceptable methods for such data collection $\mathrm{X}$-ray, CT, and MRI[14]. The analysis of the accumulated data confirms that comparison of the images taken from the same area by X-ray and MRI can confirm the cartilage grows[15]. 
Below we will connect contemporary observations of cervical cartilage degradation $\Theta$ recovery equilibrium[16] with the ideas of homeostasis[17] from the point of view of biological applications of the thermodynamics of irreversible processes [18-20].

\section{Formulation of the problem}

Contemporary thermodynamics of irreversible processes suggests that every living creature reminds a surfer of the energy flow, and is called a dissipative structure[19, 20]. These structures require somehow organized feedback, that can adjust variations in different parameters to keep the structure in certain frames. In Chemistry it causes self-oscillation like Belousov[21] or Sel'kov [22] reactions. Namely this way of organization of dissipative structure looks for the bystander as homeostasis of living creature. We need to underline, that homeostasis is maintainable only if parameters do not cross the critical borders that are not allowed the system (dissipative structure) to return back to the realm where it still can surf [23]. In biological systems the adjustment is centralized. The supreme center of the regulation for vertebrae is located in the brain[24]. And now we would like to understand if we were able to treat the patient's herniated cervical cartilage the way that everything starts to work properly, how we can prove that homeostasis will no longer let cartilage leave the borders of degradation $\Theta$ recovery equilibrium.

\section{The hypothesis}

We hypothesize that arterial hypertension (AHT) appears as a reaction of oxygen detectors in the brain on the decrement of the oxygen availability in the blood flow to the detector because of the jam, caused by the cervical cartilage damage and the cascade of the processes described extensively below. This cascade resulted in the elevation of blood pressure (BP).

A concept of centralized aerobic (AE) and anaerobic (AN) energy balance compensation CAAEBC of the human body proposed, that to maintain a constant level of (ECONST) energy metabolism in the brainstem, the microcirculatory and cellular levels of the $\mathrm{AE}$ (oxygen - EAE) and AN (glucose, lipoproteins, etc. - EAN) molecular components of the metabolism are constantly monitored by special brain centers to fulfill

$$
\mathrm{ECONST}=\mathrm{E}_{\mathrm{AE}}+\mathrm{EAN}_{\mathrm{AN}}
$$

The decrease of EAE, due to particular reasons (reduction in oxygen content in the microcirculatory bed and brain stem cells), two types of centralized adaptation reactions take place in order to maintain the overall unchanged ECONST value. These are reactions of CAAEBC in order to maintain the unchanged level of Econst. Moreover, the reactions of AN compensation, as less energy-efficient[25], are triggered only with the complete depletion of the reserves of $\mathrm{AE}$ compensation reactions. For the very schematic representation of this energy flow, AE compensation reactions are neurogenic cardiovascular reactions, which are expressed in a steady rise in BP (an increase in the cardiac output force), a narrowing of the peripheral capillaries at rest, and an increase in cardiac rate. The goal of the $\mathrm{AE}$ compensation reaction is to increase the brain stem blood perfusion and hence restoration of the EAE level.

AN compensation reactions are neurohumoral metabolic reactions that lead to an increase in the AN metabolism of sugars, phospholipids, and other energetically rich biochemical compounds. The purpose of this reaction is to increase EAN, to maintain the balance of ECONST, with a reduced EAE.

According to Dobroborskiy such reactions of the organism are manifestations of phenotypic adaptation (PA) [26]. The PA of a living organism to any changes in the environment first takes place by small forces along a simpler path. First of all, oxygen saturation of the brain occurs to a certain level, after which the reflex mechanism causing compensatory AHT is disconnected. If the brain encounters oxygen starvation for an extended period of time, then according to PA theory, changes occur at the biochemical level, namely, 
the balance of biochemical process shifts, that is, the contribution to the energy balance of AN processes increases and the contribution of AE processes decreases.

The brain, lacking oxygen, determines its decrease, as a decrease in the level of oxygen in the atmosphere and thus, tries to adapt the work of the organism under an $\mathrm{AE}$ conditions [27, 28]. In other words, the brain tries to adapt to the already changed, according to the information from the oxygen detector, external environment, which in fact has remained the same. Since the brain in such a situation begins to receive signals about the critical conditions of the heart, then, as a control center, in order to save the cardiac resource, it rebuilds the biochemical processes under conditions of a reduced partial pressure of oxygen[29]. There is a shift in $\mathrm{AE} \Theta \mathrm{AN}$ equilibrium towards $\mathrm{AN}$, thus preserving the overall balance of energy that is necessary to fulfill Bauer's universal principle of biology[30] and to balance the effect on the body of the second law of thermodynamics.

Considering the work of these compensatory mechanisms - "fast" and "slow", we could present a remarkable clinical example - squeezing the vessels of the neck to cause a person short-term hypoxia (oxygen deprivation) or tough physical exercise (sudden increment of oxygen consumption) will immediately result in the reflexive increase of his $\mathrm{BP}$ and heart rate[27, 28]. When the squeezing or exercise ends, all vital signs will quickly recover back to normal. This is an example of "quick" adaptation.

If a person already has a long-term occlusion of the vessels due to cervical osteochondrosis or there is a narrowing of the lumen of the vessels due to the atherosclerotic process, we will see the manifestations of the action of the "slow" adaptation with a shift in the $\mathrm{AE} \Theta \mathrm{AN}$ balance, namely, the development of the metabolic syndrome in general, and the type 2 diabetes[31], in particular.

Let's see how such hypothesis allows to predict the work of the behavior of cervical cartilage discs and what measurements should be presented to justify it.

\section{Discussion on the hypothesis verification}

Let's continue with AHT, caused by intervertebral discs compression with hernias and protrusions of the cervical spine. The anatomical features of the cervical vertebrae are such that veins and arteries pass through the holes in their transverse processes (arteria vertebralis, venae vertebralis). Due to the displacement of the vertebrae and the constant deep muscle spasm around them, the vessels are subsequently clamped, their lumen is narrowed (the arteries are thin and convoluted), and the blood flow is reduced up to ten times or may even be stopped at all and, as a result, the amount of delivered oxygen to the oxygen detectors in the brain is dramatically reduced. Since the brain through detectors signal observes the lacks oxygen, it takes emergency measures and commands the heart to increase the strength and/or heart rate so that blood, through all the blocks and obstacles, is still able to reach the brain and provide much needed oxygen. A stable compensatory increase in pressure and/or heart rate is being developed - thus the brain is protected from hypoxia. Therefore, as soon as it is possible to unlock the vertebral arteries and veins, the pressure and heart rate should return to the normal[27, 28]. In this case, if we are able to heal the patients from AHT through the restoration of vertebral arteries patency, and confirm that cervical spine cartilage discs are developing toward restoration, then our hypothesis should be considered as confirmed. The normalization of AHT through the restoration of the blood flow of the brain stem is easy to register by measurement of $\mathrm{BP}$ and arterial linear blood flow $\left(\mathrm{V}_{\mathrm{A}}\right)$ (This preliminary data is collected and exhibited in Table 1). 
Table 1. Results on the restoration of vertebral arteries $V_{A}$ by cervical cartilage recovery according to [32]. The data was collected for the entire 2019 from all patients who attended our clinic with AHT diagnosis (total of 2622).

\begin{tabular}{ccc}
\hline Parameter & Patients number & \% of total \\
\hline Lowering BP on 10-20 torr & $740^{*}$ & 28 \\
Lowering BP on 20-40 torr & $1604^{*}$ & 61 \\
Lowering BP for $\geq 40$ torr & $278^{*}$ & 11 \\
Pulse normalization & 2342 & 89 \\
Increment of $\mathrm{V}_{\mathrm{A}} \geq 25 \%$ & 2622 & 100 \\
\hline
\end{tabular}

- $\quad-740+1604+278=2622$, so the BP lowering experienced $100 \%$ of the patients

In this case the decompression of the vertebral arteries and veins during the correction of deep neck muscles leads to measurable restoration of $\mathrm{V}_{\mathrm{A}}$ of sinistra and dextra arteria vertebralis to the values of arteria carotis communis. The fact is that with age, there is a progression of osteochondrosis and complications arise that are associated with the gradual displacement of the cervical vertebrae. To prove this hypothesis, we will need to compare data before and after treatment. (This preliminary data is collected, but not shown)

As a rule, in most elderly people, the diagnosis of "essential" or "idiopathic" AHT simply implies nothing more than a compensatory increase in BP due to circulatory disturbances in the brain stem due to compression of the vessels at the level of the cervical spine[33-36].

Over time a patient develops an osteochondrosis process in the cervical region, and since this is where the vertebral arteries pass through the transverse processes of the vertebrae, their lumen naturally narrows blood flow into the brain stem and into the rhomboid fossa where the vascular center is located. Osteochondrosis is a disease that is directly related to the psychological state of the individual, namely, the accumulation of stress factors in the body. This happened evolutionarily so that any nervous shock manifests itself in the tension of the neck muscles. Therefore, it is necessary to "squeeze the head in the shoulders, hide it" to protect the cervical arteries, because the predator, attacks the neck first. At its core, this reaction is atavism. Evolution should continue and similar atavisms will eventually disappear. But while there is such an effect, any stress, social or any other kind, produces an automatic reaction - the neck strains, and the head is drawn into the shoulders. Most people have special target muscles that harden when stressed[37]. This is a kind of vicious circle: stress causes the release of adrenaline, which in turn strains the muscles of the neck and upper back, making even more adrenaline, etc.

Soon enough this condition becomes habitual. There is a chronic spasm of deep muscles and in general a spasm of all muscles of the cervical and thoracic sections. Against this background the microcirculation and nutrition of the intervertebral discs and ligaments is disturbed, their weakening occurs, and the vertebrae begin to shift and clamp the vessels. The partial pressure of oxygen in the brain stem decreases because the flow of blood decreases. Due to the activation of the vascular center in the rhomboid fossa, the brain stem delivers an efferent signal to the heart and it starts increasing the pressure by increasing the heart force and the heart rate. According to Dobroborskiy's theory, this is the first stage of PA. According to the CAAEBC, it is an AN compensation of the energy balance.

That is to say that the central apparatus - the brain - regularly receives less oxygen than it should have received by the parameters that are genetically set as a normal[38]. Accordingly, the control center proceeds to the processes of additional energy release from processes not related to breathing (AN).

The brain marks regular overloads and strains on the heart muscle and then activates the mechanism of spasm of the peripheral vessels, thus reducing the burden on the heart. 
Gradually, a slow centralization of blood circulation takes place. Therefore, the brain temporarily compensates for the state of hypoxia. With the unjustified administration of betablockers and vasodilators acting on the renin-angiotensin-aldosterone system, the brain receives an additional toxic effect that nullifies all its adaptive responses. The control center persistently continues the process of compensating the energy balance, due to which, over time, these drugs cease to function properly[39-41].

When the process of oxygen starvation is aggravated, the following adaptive mechanism is activated: the biochemical component. There is a need for extracting energy from AN source. There is a shift in AE $\leftrightarrow$ AN energy balance, and then a shift in the acid-base balance, resulting in the body acidosis[42-44]. Thus, the picture of PA related to functioning in a medium without oxygen is traced. It looks, that diabetes mellitus type 2 could be developed this way in the form of impaired insulin metabolism and disruption of the normal functioning of the pancreas. That is, the organism tries to preserve the energy constant in the body with the help of these reactions.

If the brain stem has already reorganized the regulation of biochemical processes due to a lack of oxygen, then it must make sure that we regularly replenish the oxygen level, and that this process will keep going to complete recovery.

\section{Conclusion}

How is it possible to prove this hypothesis on with objective methods? In addition to the data on BP and $\mathrm{V}_{\mathrm{A}}$ we need to collect the data on blood biochemical parameters, including $\mathrm{pH}$ and $[\mathrm{Fe}]$, and geometry of the cartilage of the cervical vertebrae by X-ray and MRI before and after recovery.

Author Contributions: Conceptualization, A.Y.S., K.V.Z., and A.A.V.; methodology, A.Y.S. and B.A.G.; software, B.A.G.; validation, A.Y.S., K.V.Z., and A.A.V.; formal analysis, A.Y.S. and A.A.V.; investigation, A.Y.S., K.V.Z., and A.A.V.; resources, A.Y.S.; data curation, B.A.G.; writing-original draft preparation, A.Y.S., K.V.Z., and A.A.V..; writing-review and editing, A.Y.S., K.V.Z., and A.A.V.; visualization, A.A.V.; supervision, A.Y.S. and A.A.V.; project administration K.V.Z.; funding acquisition, A.Y.S. All authors have read and agreed to the published version of the manuscript.

Funding: This paper has been supported by the RUDN University Strategic Academic Leadership Program (recipient A.A.V.)

Conflicts of Interest: The authors declare no conflict of interest.

\section{References}

1. Choy, W.J.; et al. Annular closure device for disc herniation: meta-analysis of clinical outcome and complications. BMC Musculoskelet Disord, 2018, 19, p. 290.

2. Kerr, D.; Zhao, W.; Lurie, J.D. What Are Long-term Predictors of Outcomes for Lumbar Disc Herniation? A Randomized and Observational Study. Clin Orthop Relat Res, 2015, 473, p. 1920-1930.

3. Sophia Fox, A.J.; Bedi, A.; Rodeo, S.A. The basic science of articular cartilage: structure, composition, and function. Sports Health, 2009, 1, p. 461-468.

4. Reddy, M.S.B.; et al. A Comparative Review of Natural and Synthetic Biopolymer Composite Scaffolds. Polymers (Basel), 2021, 13.

5. Oosterhuis, T.; et al. Rehabilitation after lumbar disc surgery. Cochrane Database Syst Rev, 2014, 3,p. CD003007.

6. Spoto, M.M.; Dixon, G. .An integrated approach to the examination and treatment of a patient with chronic low back pain. Physiother Theory Pract, 2015, 31, p. 67-75.

7. Azevedo, D.C.; et al. Movement System Impairment-Based Classification Versus General Exercise for Chronic Low Back Pain: Protocol of a Randomized Controlled Trial. Phys Ther, 2015, 95, p. 1287-1294.

8. Robinson, M. Clinical diagnosis and treatment of a patient with low back pain using the patient response model: A case report. Physiother Theory Pract, 2016, 32, p. 315-323.

9. Tavakoli, J.; Diwan, A.D.; Tipper, J.L. Advanced Strategies for the Regeneration of Lumbar Disc Annulus Fibrosus. Int J Mol Sci, 2020, 21, p.4889

10. Choi, Y.; Park, M.H.; Lee, K. Tissue Engineering Strategies for Intervertebral Disc Treatment Using Functional Polymers. Polymers (Basel), 2019,11 p.872

11. Chu, G.; et al. Biomechanics in Annulus Fibrosus Degeneration and Regeneration. Adv Exp Med Biol, 2018, 1078, p. 409-420. 
12. Moriguchi, Y.; et al. In vivo annular repair using high-density collagen gel seeded with annulus fibrosus cells. Acta Biomater, 2018, 79, p. 230-238.

13. Bowles, R.D.; Setton, L.A. Biomaterials for intervertebral disc regeneration and repair. Biomaterials, 2017, 129, p. 54-67.

14. Benneker, L.M.; et al. Correlation of radiographic and MRI parameters to morphological and biochemical assessment of intervertebral disc degeneration. Eur Spine J, 2005, 14, p. 27-35.

15. Laor, T.; Clarke, J.P.; Yin, H. Development of the long bones in the hands and feet of children: radiographic and MR imaging correlation. Pediatr Radiol, 2016, 46, p. 548-561.

16. Karsdal, M.A.; et al. Cartilage degradation is fully reversible in the presence of aggrecanase but not matrix metalloproteinase activity. Arthritis Res Ther, 2008, 10, p. R63.

17. Billman, G.E. Homeostasis: The Underappreciated and Far Too Often Ignored Central Organizing Principle of Physiology. Front Physiol, 2020, 11, p. 200.

18. Tlidi, M.; et al. Observation and modelling of vegetation spirals and arcs in isotropic environmental conditions: dissipative structures in arid landscapes. Philos Trans A Math Phys Eng Sci, 2018, 376, p.2135.

19. Tlidi, M.; Clerc, M.G.; Panajotov, K. Dissipative structures in matter out of equilibrium: from chemistry, photonics and biology, the legacy of Ilya Prigogine (part 1). Philos Trans A Math Phys Eng Sci, 2018, 376, p.2124.

20. Tlidi, M.; Clerc, M.G.; Panajotov, K. Dissipative structures in matter out of equilibrium: from chemistry, photonics and biology, the legacy of Ilya Prigogine (part 2). Philos Trans A Math Phys Eng Sci, 2018, 376, p.2134.

21. Belousov, B.P. A periodic reaction and its mechanism. In: Collection of short papers on radiation medicine conference for 1958, Moscow, Russia, 1958, Med. Publ. 1959

Sel'kov, E.E.; Self-Oscillations in Glycolysis.1. A Simple Kinetic Model. European J. Biochem. , 1968, 4, p. 79-86. Metab, 2016, 24, p. 269-82.

Roh, E.; Kim, M.S. Brain Regulation of Energy Metabolism. Endocrinol Metab (Seoul), 2016, 31, p. 519-524.

Nelson, D.L.; Cox, M.M. Lehninger principles of biochemistry. 5th ed. 2008, NY: W.H.Freeman.

Dobroborsky, B.S.; Thermodynamics of Biological Systems. 2006, Saint-Petersburg, Russia: North-Western State MedicalUniversity Press.

27. Curtelin, D.; et al. Cerebral blood flow, frontal lobe oxygenation and intra-arterial blood pressure during sprint exercise in normoxia and severe acute hypoxia in humans. J Cereb Blood Flow Metab, 2018, 38, p. 136-150.

28. He, Z.B.; et al. Atlantoaxial Misalignment Causes High Blood Pressure in Rats: A Novel Hypertension Model. Biomed Res Int, 2017, 2017, p. 5986957.

29. Silvani, A.; et al. Brain-heart interactions: physiology and clinical implications. Philos Trans A Math Phys Eng Sci, 2016, 374, p. 2067.

30. Elek, G.; Muller, M. The living matter according to Ervin Bauer (1890-1938), (on the 75th anniversary of his tragic death) (History). Acta Physiol Hung, 2013, 100, p. 124-132.

31. Levit, S.; et al. Type 2 diabetes therapeutic strategies: why don't we see the ELEPHANT in the room? Diabetes Mellitus, 2016, 19, p. 341-349.

32. Shishonin, A. Method for treating cervical osteochondrosis., p.a.t. Russian Federation Federal Service for intellectual property, Editor. 2003: Russian Federation.

33. Gwadry-Sridhar, F.H.; et al. Impact of interventions on medication adherence and blood pressure control in patients with essential hypertension: a systematic review by the ISPOR medication adherence and persistence special interest group. Value Health, 2013, 16, p. 863-871.

34. Salvi, E.; et al. Genomewide association study using a high-density single nucleotide polymorphism array and case-control design identifies a novel essential hypertension susceptibility locus in the promoter region of endothelial NO synthase. Hypertension, 2012, 59, p. 248-255.

35. Morgan, T.O.; Anderson, A.; Bertram, D.Effect of indomethacin on blood pressure in elderly people with essential hypertension well controlled on amlodipine or enalapril. Am J Hypertens, 2000, 13, p. 1161-1167.

36. Hoffmann, J.; et al. European headache federation guideline on idiopathic intracranial hypertension. J Headache Pain, 2018, 19 , p. 93.

37. Kroll, L.S.; et al. Level of physical activity, well-being, stress and self-rated health in persons with migraine and co-existing tension-type headache and neck pain. J Headache Pain, 2017, 18, p. 46.

38. De Backer, J.; et al. A reliable set of reference genes to normalize oxygen-dependent cytoglobin gene expression levels in melanoma. Sci Rep, 2021, 11,: p. 10879.

39. Lipworth, B.; et al. Beta-blockers in COPD: time for reappraisal. Eur Respir J, 2016, 48, p. 880-888.

40. Fu, M. Beta-blocker therapy in heart failure in the elderly. Int J Cardiol, 2008, 125, p. 149-153.

41. Couffignal, C.; et al. Timing of beta-Blocker Reintroduction and the Occurrence of Postoperative Atrial Fibrillation after Cardiac Surgery: A Prospective Cohort Study. Anesthesiology, 2020, 132, p. 267-279. 
43. Regolisti, G.; et al. [Metabolic acidosis]. G Ital Nefrol, 2016, 33.

44. Sajan, A.; et al. Recurrent Anion Gap Metabolic Acidosis. Am J Med Case Rep, 2019, 7, p. $200-202$. 